\title{
Identificação dos principais autores em planejamento e controle da produção por meio de um survey mundial com pesquisadores da área
}

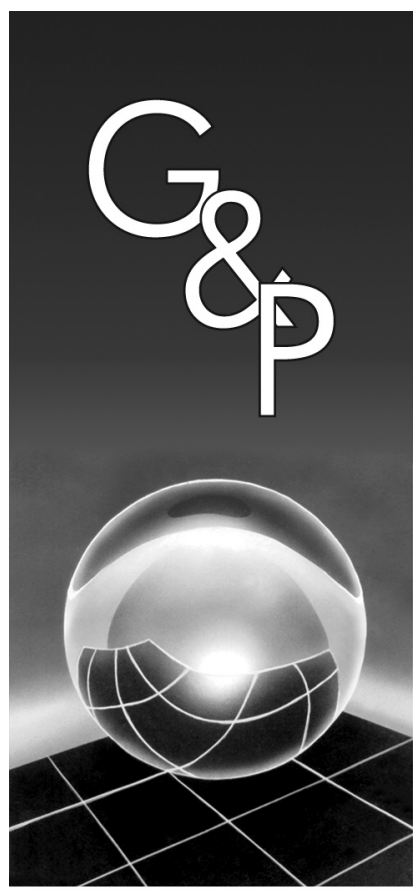

\author{
Flávio Cesar Faria Fernandes \\ Fábio Azeka \\ Maria Cecília Mendes Barreto \\ Moacir Godinho Filho
}

\section{Resumo}

O planejamento e controle da produção (PCP), apesar de ser uma área antiga e possuir uma enorme quantidade de pesquisas já desenvolvidas, ainda não tem um consenso sobre quem são os seus principais autores. O objetivo deste trabalho é identificar os principais autores da área PCP por meio de um survey mundial com pesquisadores da área. Foram identificados os dez principais autores na opinião dos pesquisadores entrevistados, bem como a influência (direta ou indireta ou sobre algum problema específico do $\mathrm{PCP}$ ) e o impacto (na indústria ou na teoria e na pesquisa ou no ensino do $\mathrm{PCP}$ ) dos principais autores. As principais contribuições deste artigo são: i) detectar, dentre uma infinidade de obras relacionadas ao PCP, quais são as de maior impacto para a área; ii) direcionar os estudos daqueles que têm interesse em aprofundar seus conhecimentos na área de PCP por meio da apresentação dos principais livros e autores da área; e iii) valorizar os principais autores da área e dar o primeiro passo na determinação dos grandes mentores ( "gurus") do PCP.

Palavras-chave: Planejamento e controle da produção. Principais autores. Survey mundial.

\section{Introdução e motivação do trabalho}

Buffa e Sarin (1987) definem sistema produtivo como o meio pelo qual recursos de entrada (input) são transformados em bens e serviços úteis como saída (output). Para Sipper e Bulfin (1997), um sistema de produção é qualquer coisa que transforma um input em um output com valor agregado. Ainda de acordo com estes autores, os sistemas de produção são proeminentes na sociedade moderna. Tais sistemas formam a base para a construção e o aumento da força econômica e vitalidade de um país. Deste modo, uma área que tem se tornado cada vez mais importante para as empresas é o planejamento e controle da produção (PCP), pois ela gerencia o fluxo de materiais do sistema de produção, por meio do fluxo de informações e decisões.

Segundo Bonney (2000), a função Planejamento e Controle da Produção e seus sistemas associados tem o objetivo de planejar e controlar a produção de forma que a empresa atinja os requisitos de produção do modo mais eficiente possível. Para Slack et al. (2002) o PCP tem o propósito de garantir que a produção ocorra eficaz e eficientemente e produza produtos e serviços conforme requeridos pelos consumidores. Isto requer que os recursos produtivos estejam disponíveis: na quantidade adequada; no momento adequado; e no nível de qualidade adequado. De acordo com Correa et al. (2001) o planejamento e controle da produção envolve uma série de decisões com o objetivo de definir o que, quanto e quando produzir e comprar, além dos recursos a serem utilizados.

Apesar da área de PCP ser antiga (Taylor e Gantt podem ser considerados os pioneiros) e possuir uma enorme quantidade de pesquisas já desenvolvidas (mais de 150000 artigos relacionados na base de dados COMPENDEX (http://www.engineeringvillage2.org) nos últimos 30 anos), ainda não existe um consenso entre os pesquisadores da área sobre quem são os principais autores do PCP, ao passo que em outras áreas, como na Gestão da Qualidade, tal consenso já existe.

Comparando superficialmente a área de Gestão da Qualidade que possui um consenso sobre seus principais 
nomes, com a área de PCP que não possui tal consenso, nota-se que a existência de grandes mentores ("gurus") contribui de uma forma muito importante para a valorização e divulgação de uma área. Além disso, uma lista com os principais autores pode ser de grande ajuda para a orientação dos estudos daqueles que têm interesse em aprender mais sobre o assunto, bem como pode ser útil para auxiliar a propagar as idéias da área.

Huczynski (1993) realizou uma pesquisa com o intuito de analisar os principais autores na área de administração. Nesse trabalho, ele verificou que os anos 80 geraram um grande interesse em idéias gerenciais e grandes mentores, mas durante este tempo, apenas um pequeno número de especialistas em administração atingiu o status de grande mentor. Segundo Huczynski (1993), uma pessoa se torna um grande mentor quando suas idéias se transformam em idéias populares. Entretanto, ele constatou que para que as idéias se popularizem elas devem passar por uma espécie de funil que contenha 5 filtros finos. Cada um desses filtros tem o seguinte rótulo: necessidade dos gerentes, benefícios da idéia, oportunidade, promoção e apresentação. Além disso, Huczynski (1993) afirma que as idéias também precisam atender a 5 requisitos: i) ser oportuna (ou seja, ela deve estar de acordo com os problemas da época); ii) ter conseguido a atenção de sua audiência potencial (as idéias não se auto promovem; universidades, consultorias, treinamentos e empresas têm um importante papel na disseminação das idéias); iii) atender aos requisitos da organização de um modo que esteja de acordo com as necessidades individuais e as preocupações do gerente, a quem ela está endereçada; iv) possuir ingredientes essenciais que permitam aos usuários potenciais perceberem que ela é relevante para que eles satisfaçam suas necessidades; e v) ser verbalmente apresentada de um modo atrativo.

Embora a pesquisa de Huczynski (1993) tenha sido voltada especificamente para a área de Administração, ela serviu para destacar a importância dos grandes mentores de uma forma geral. Segundo o autor, quando uma pessoa se torna um grande mentor, a sua obra é imortalizada e, mesmo que ela se torne ultrapassada e, posteriormente, outros autores refutem suas idéias, ela ainda será uma referência para a área, assim, dentre outras coisas, no mínimo ela servirá para que as pessoas entendam o processo de evolução das principais idéias da área. Como exemplo tem-se o caso de Taylor que escreveu seu livro há um século atrás e que, apesar de algumas de suas idéias estarem "fora de moda" atualmente, ainda é uma referência.

Um ponto que merece ser destacado é o fato de Huczynski (1993) afirmar que o livro é o ponto inicial para a promoção de um grande mentor, pois, se o livro for um sucesso de vendas (best-selller), ele dará ao autor a atenção de uma grande audiência.
Assim, este trabalho pretende dar um primeiro e importante passo para a identificação dos grandes mentores da área de PCP pela identificação dos autores dos livros mais importantes na visão de uma amostra mundial de pesquisadores em PCP. Espera-se que a elaboração de uma lista com os principais autores da área de PCP e seus livros, possa servir como base para o direcionamento de outras pesquisas, bem como poderá facilitar os estudos sobre PCP para todos aqueles que tiverem interesse pelo assunto.

A estrutura do trabalho é a que segue: na seção 2, são apresentadas formalmente as questões de pesquisa que este trabalho pretende responder, bem como as hipóteses e objetivos do trabalho; na seção 3, é apresentada a metodologia de pesquisa utilizada no trabalho, bem como os passos da pesquisa; na seção 4, são apresentados os passos da pesquisa; na seção 5, são mostrados os resultados da pesquisa; na seção 6 , estes resultados são analisados, bem como as hipóteses levantadas são avaliadas; e na seção 7, são tecidas algumas considerações finais.

\section{Objetivos do trabalho e hipóteses a serem testadas}

As principais questões de pesquisa que norteiam o presente trabalho são:

a) quem são os principais autores de PCP segundo pesquisadores internacionais da área?; e

b) quais são os principais livros escritos pelos autores do PCP identificados na questão 1 ?

Portanto o principal objetivo desta pesquisa é identificar os principais autores de PCP segundo a visão de acadêmicos em nível mundial, bem como suas obras. Além disso, pretende-se também identificar a influência dos principais autores para a área de PCP (influência direta sobre a área; influência sobre um problema específico de PCP; ou influência indireta sobre o PCP) e também o impacto do autor sobre a área (impacto na teoria e pesquisa sobre PCP; impacto no ensino de PCP; ou impacto na indústria e prática do $\mathrm{PCP}$ ).

Também foram levantadas algumas hipóteses as quais tem como propósito embasar o objetivo principal desta pesquisa. Estas hipóteses são as seguintes:

a) hipótese 1: os pesquisadores, de forma geral, tendem a escolher autores relacionados ao seu enfoque de pesquisa;

b) hipótese 2: existe certo "regionalismo" entre os pesquisadores internacionais, ou seja, pessoas formadas em uma dada região (por exemplo, Europa) tendem a estudar e, conseqüentemente, a votar em autores da própria região; $\mathrm{e}$

c) hipótese 3: o fato de um autor participar da elaboração de um livro clássico e bem conceituado, que pode ser considerado como um dos livros mais 
importantes da área de PCP, não garante que o autor possa ser classificado como um dos principais autores da área.

\section{Metodologia de pesquisa}

Dentre os vários procedimentos de pesquisa, devido aos seus objetivos, este trabalho se utiliza do survey. $\mathrm{O}$ survey consiste de uma série de questionários ou entrevistas estruturadas, com o objetivo de examinar padrões e relacionamentos entre as variáveis de estudo (BRYMAN, 1995). Neste procedimento, não existe a intervenção do pesquisador no experimento. Os dados conseguidos por meio do survey devem ser passíveis de análise estatística. De acordo com Creswell (1994), o survey visa proporcionar uma descrição numérica de alguma forma, sendo, portanto, um procedimento quantitativo.

Para a realização do survey de forma a cumprir os objetivos do presente trabalho, bem como validar ou não as hipóteses mencionadas, a presente pesquisa segue a seguinte seqüência de passos:

- Passo 1: Determinação dos critérios para que um pesquisador pudesse fazer parte da população de pessoas aptas a participarem da pesquisa (determinação da população);

- Passo 2: Coleta inicial de dados e identificação das principais características da população de pesquisadores;

- Passo 3: Determinação da amostra de pesquisadores;

- Passo 4: Verificação se a amostra possui basicamente as mesmas características da população de pesquisadores;

- Passo 5: Determinação dos critérios de inclusão de um autor na lista dos principais autores e de uma lista inicial de top-autores;

- Passo 6: Determinação da técnica para realizar a votação com a amostra de pesquisadores selecionados e elaboração do questionário;

- Passo 7: Envio do questionário por e-mail a todos os pesquisadores da amostra selecionada e recebimento e armazenamento das respostas. Nesta etapa, também foi realizada uma preparação dos dados para o passo seguinte; e

- Passo 8: Apresentação e análise estatística dos resultados .

Nas próximas seções cada um destes passos é pormenorizado.

\section{Os passos da pesquisa}

Nesta seção, são apresentados os passos de 1 a 7 da presente pesquisa.
Passo 1: Critérios para a inclusão dos pesquisadores que estariam aptos a participarem da pesquisa.

Houve uma atenção especial com a escolha desses critérios, procurando minimizar os possíveis vieses para a formação da população, visto que um erro nesta etapa poderia comprometer todo o restante da pesquisa.

Critérios para fazer parte da população:

a) ser pesquisador da área de planejamento e controle da produção;

b) o pesquisador deve ter pelo menos um artigo publicado em inglês, na área de PCP, de janeiro de 1999 a dezembro de 2000, nos principais periódicos ligados ao PCP: Computers \& Industrial Engineering; IIE Transactions; Interfaces; International Journal of Operations \& Production Management; International Journal of Production Economics; International Journal of Production Research; Journal of Manufacturing Systems; Journal of Operations Management; Management Science; Naval Research Logistics; Omega; Operations Research/ Management Science; Production and Inventory Management; Production and Operations Management; Production Planning \& Control, entre outros; e

c) o pesquisador deve possuir um e-mail que esteja disponibilizado pelos periódicos ou pela Internet via sites de busca.

Um total de 592 pesquisadores atendeu esses critérios e eles formaram a população do estudo.

Passo 2: Coleta inicial de dados e identificação das principais características da população de pesquisadores.

Para cada pesquisador da população, obtiveram-se inicialmente os seguintes dados: i) nome do pesquisador; ii) título acadêmico; iii) e-mail; iv) universidade ou instituição; v) departamento; vi) país; vii) região mundial (Europa, Estados Unidos ou Resto do Mundo); e vii) principais enfoques de sua pesquisa ou trabalho.

Com relação aos enfoques de pesquisa estes foram divididos em quatro classes: Aspectos Gerenciais (conceitos, procedimentos e práticas gerenciais) de PCP, aplicações de pesquisa operacional em PCP, aplicações de inteligência artificial em PCP e aplicações de sistema de informação/tecnologia de informação em PCP.

Foram levantadas as principais características da população de pesquisadores, a fim de posteriormente serem comparadas às características da amostra. Estas características são: heterogeneidade geográfica e heterogeneidade quanto ao número de publicações individuais.

a) Heterogeneidade geográfica.

A população apresentou uma grande heterogeneidade geográfica visto que ela é formada por 47 países. A Tabela 1 mostra todos os países integrantes da população e suas respectivas quantidades e porcentagens de pesquisadores. Do total de 592 pesquisadores, 180 são 
Tabela 1. Distribuição geográfica dos pesquisadores da população.

\begin{tabular}{|c|c|c|c|c|c|c|c|}
\hline $\mathbf{N}^{0}$ & País & $\mathrm{N}^{\circ}$ Pesq. & Pesq. (\%) & $\mathbf{N}^{0}$ & País & $\mathrm{N}^{\circ}$ Pesq. & Pesq. (\%) \\
\hline 1 & EUA & 223 & 37,67 & 25 & Kuwait & 4 & 0,68 \\
\hline 2 & Inglaterra & 29 & 4,90 & 26 & Polônia & 4 & 0,68 \\
\hline 3 & Alemanha & 28 & 4,73 & 27 & Suíça & 4 & 0,68 \\
\hline 4 & Japão & 26 & 4,39 & 28 & Portugal & 3 & 0,51 \\
\hline 5 & França & 26 & 4,39 & 29 & Tailândia & 3 & 0,51 \\
\hline 6 & Holanda & 23 & 3,89 & 30 & Nova Zelândia & 3 & 0,51 \\
\hline 7 & Itália & 22 & 3,72 & 31 & Argentina & 2 & 0,34 \\
\hline 8 & Coréia & 21 & 3,55 & 32 & Áustria & 2 & 0,34 \\
\hline 9 & Canadá & 20 & 3,38 & 33 & Bahrein & 2 & 0,34 \\
\hline 10 & China & 16 & 2,70 & 34 & Bielo Rússia & 2 & 0,34 \\
\hline 11 & Taiwan & 14 & 2,36 & 35 & Eslovênia & 2 & 0,34 \\
\hline 12 & Hong Kong & 13 & 2,20 & 36 & Espanha & 2 & 0,34 \\
\hline 13 & Turquia & 13 & 2,20 & 37 & Líbano & 2 & 0,34 \\
\hline 14 & Singapura & 10 & 1,69 & 38 & Chile & 1 & 0,17 \\
\hline 15 & Índia & 9 & 1,52 & 39 & Croácia & 1 & 0,17 \\
\hline 16 & Israel & 9 & 1,52 & 40 & Dinamarca & 1 & 0,17 \\
\hline 17 & Suécia & 8 & 1,35 & 41 & Emirados A. Unidos & 1 & 0,17 \\
\hline 18 & Brasil & 7 & 1,18 & 42 & Indonésia & 1 & 0,17 \\
\hline 19 & Bélgica & 6 & 1,01 & 43 & Marrocos & 1 & 0,17 \\
\hline 20 & Arábia Saudita & 5 & 0,84 & 44 & Noruega & 1 & 0,17 \\
\hline 21 & Austrália & 5 & 0,84 & 45 & Porto Rico & 1 & 0,17 \\
\hline 22 & Finlândia & 5 & 0,84 & 46 & Romênia & 1 & 0,17 \\
\hline 23 & Grécia & 5 & 0,84 & 47 & Rússia & 1 & 0,17 \\
\hline 24 & Hungria & 4 & 0,68 & & Total & 592 & 100 \\
\hline
\end{tabular}

da Europa, 223 dos Estados Unidos e 190 do resto do mundo.

b) Heterogeneidade quanto ao número de publicações individuais.

A heterogeneidade quanto ao número de publicações individual é grande, uma vez que existe uma variação de um a nove artigos publicados por pessoa num período de dois anos (Tabela 2).

Passo 3: Determinação da amostra de pesquisadores.

O tamanho da amostra aleatória simples foi calculado utilizando a fórmula de determinação para populações pequenas (inferiores a 100.000), mostrada em Rea e Parker (1997) e Burns e Bush (2000), que é expressa por:

$$
n=\frac{Z_{\alpha}^{2}(0,25) N}{Z_{\alpha}^{2}(0,25)+(N-1) C_{p}^{2}}
$$

sendo $Z_{\alpha}$ : quantil $1-(\alpha / 2)$ de uma distribuição normal padrão; $N$ : tamanho da população; e $C_{p}$ : margem de erro entre o valor estimado e o valor verdadeiro.

Adotou-se 95\% de nível de confiança (o que resulta em um $\left.Z_{95}=1,96\right)$ e intervalo de confiança de 5,5\%. Assim, para um tamanho da população de 592 tem-se uma amostra de aproximadamente 205. Este foi o tamanho de amostra determinado.

Resolveu-se ainda que pesquisadores com maior número de artigos publicados deveriam ter uma probabilidade maior de serem sorteados para compor a amostra. A ponderação adotada é mostrada na Tabela 3.
Tabela 2. Distribuição de pesquisadores da população em função do número de publicações.

\begin{tabular}{ccc}
\hline No de publicações & \multicolumn{2}{c}{ População } \\
\cline { 2 - 3 } & $\mathbf{N}^{\mathbf{0}}$ pesq. & Pesq. (\%) \\
\hline 1 & 420 & 70,95 \\
2 & 122 & 20,61 \\
3 & 38 & 6,42 \\
4 & 8 & 1,35 \\
5 & 1 & 0,17 \\
6 & 1 & 0,17 \\
8 & 1 & 0,17 \\
9 & 1 & 0,17 \\
Total & 592 & 100 \\
\hline
\end{tabular}

Tabela 3. Atribuição dos pesos de seleção segundo o número de publicações.

\begin{tabular}{cc}
\hline $\mathbf{N}^{\mathbf{0}}$ publicações & Peso $\boldsymbol{M}_{\boldsymbol{i}}$ \\
\hline 1 & 1 \\
2 & 2 \\
3 & 3 \\
4 & 3 \\
5 & 4 \\
6 & 4 \\
8 & 4 \\
9 & 4 \\
\hline
\end{tabular}


A atribuição de diferentes pesos de seleção aos pesquisadores conduz ao uso do processo de amostragem com probabilidades desiguais (desenvolvido por Hansen e Hurwitz (1943) e abordado por Cochran (1977)), o qual considera $p_{i}$ a probabilidade de seleção de um particular elemento da população. Essas probabilidades são proporcionais aos pesos dados na Tabela 3. O método cria para cada pesquisador $(i=1,2, \ldots, N)$ um intervalo de seleção $\left(I S_{i}\right)$ proporcional ao valor do peso $M_{i}$. Assim, após conhecer todos os valores de $M_{i}$ deve-se formar uma coluna $\sum_{i=1} M_{i}$ que é o valor da somatória acumulada dos

pesos. Com esses elementos formam-se os intervalos de seleção $\left(I S_{i}\right)$, utilizando o seguinte procedimento:

a) O primeiro intervalo de seleção $I S_{I}(i=1)$ inicia-se com o valor 1 e termina com o valor de $M_{i}$; e

b) Os outros intervalos são formados por $\left(\sum_{i=1}^{j-1} M_{i}, \sum_{i=1}^{j} M_{i}\right]$, ou seja, para o indivíduo $j$ inicia-se pelo valor aberto de $\sum_{i=1}^{j-1} M_{i}$ e termina-se com o valor fechado de $\sum_{i=1}^{j} M_{i}$ para $j=2, \ldots, \mathrm{N}$.

Assim, forma-se a Tabela 4, na qual é calculado o intervalo de seleção para cada pesquisador a partir dos respectivos valores da soma acumulada dos pesos $M_{i}$.

Depois de concluído o cálculo de todos os intervalos de seleção, utilizou-se o gerador de números pseudoaleatórios do Microsoft ${ }^{\circledR}$ Excel para selecionar a amostra. Por fim, organizaram-se os 205 nomes obtidos em uma tabela.

Cabe ressaltar que até o presente momento não existe uma fórmula específica para a determinação do tamanho de amostra aleatória com probabilidades diferentes. Por isso optou-se por usar a fórmula para amostragem aleatória simples.
Passo 4: Verificação se a amostra possui basicamente as mesmas características da população de pesquisadores.

Foram comparadas as características heterogeneidade geográfica e heterogeneidade quanto ao número de publicações para verificar se a amostra obtida tem basicamente as mesmas características da população de pesquisadores.

Da mesma forma que a população, a amostra de pesquisadores também possui uma grande heterogeneidade geográfica visto que ela é composta por 205 pesquisadores divididos em 37 países. Verificou-se também, por meio de tabelas, que a amostra preservou, em relação à população, a heterogeneidade quanto ao número de publicações por pesquisador.

Passo 5: Determinação dos critérios de inclusão de um autor na lista dos principais autores e de uma lista inicial de top-autores

Antes de se iniciar a pesquisa propriamente dita, foram estabelecidos critérios para uma pessoa ser candidata à lista dos top-autores. Estes critérios são: a) o autor apontado (pode estar vivo ou morto) deve ter escrito um livro clássico da área de PCP (não importa se o livro é atual ou não, o importante é que tenha trazido uma real contribuição para a área); e b) ser um pesquisador respeitado pelas suas publicações nos periódicos da área ou um autor respeitado pelos profissionais que trabalham em PCP em empresas.

Para se tentar minimizar uma possível alta taxa de não resposta na pesquisa, foram levantados inicialmente alguns importantes autores para que estes servissem de base para a pesquisa. Neste trabalho inicial, foram enviadas questões para 60 pesquisadores (20 da Europa, 20 dos Estados Unidos e 20 do resto do mundo) com o objetivo de determinar os top-autores na visão deles. Ao final, chegou-se às seguintes conclusões:

a) a receptividade da pesquisa foi muito grande, 26 respostas em 60 e-mails (43\%) sem termos replicados, e sem termos preocupados em justificar a

Tabela 4. Modelo da tabela com os cálculos dos intervalos de seleção dos pesquisadores da população.

\begin{tabular}{rlcccc}
\hline $\mathbf{N}^{\mathbf{0}}$ & Nome & $\mathbf{N}^{\mathbf{0}}$ publicações & Peso $\boldsymbol{M}_{\boldsymbol{i}}$ & $\sum \boldsymbol{M}_{\boldsymbol{i}}$ & Intervalo seleção \\
\hline 1 & Abad, P. L. & 1 & 1 & 1 & $1-1$ \\
2 & Abboud, N. E. & 1 & 1 & 2 & $2-2$ \\
$\ldots$ & $\ldots$ & $\ldots$ & $\ldots$ & $\ldots$ & $\ldots$ \\
542 & Wan, Y.-W. & 1 & 1 & 745 & $745-745$ \\
543 & Wang, D. & 4 & 3 & 748 & $746-748$ \\
544 & Wang, R.-C. & 1 & 1 & 749 & $749-749$ \\
545 & Wang, W. & 3 & 3 & 752 & $750-752$ \\
546 & Wang, Y. & 2 & $\ldots$ & 754 & $753-754$ \\
$\ldots$ & $\ldots$ & $\ldots$ & 1 & $\ldots$ & $\ldots$ \\
591 & Zuo, M. J. & 1 & 1 & 813 & $813-813$ \\
592 & Zydiak, J. L. & 1 & & \\
\hline
\end{tabular}


pesquisa. E muitos solicitaram o nome do livro que fora escrito por cada um dos autores sugeridos na lista;

b) nos primeiros e-mails recebidos, nenhum autor foi indicado consensualmente;

c) à medida que as pessoas respondiam, acrescentavam sugestões de novos nomes, que foram incorporados aos já sugeridos, nos e-mails seguintes;

d) observou-se que as questões abertas tinham um nível de não-resposta muito alto, enquanto que as questões mais fáceis de responder (questões fechadas) conseguiam um maior índice de respostas; e

e) ao final da pesquisa obteve-se uma lista inicial dos top-autores do PCP com 31 nomes: Bailey, J. E.; Baker, K.; Bedworth, D. D.; Berry, W. L.; Billington, P.; Buffa, E. S.; Bulfin, R.; Burbidge, J. L.; Elmaghraby, S. E.; Goldratt, E.; Hax, A. C.; Hopp, W. J.; Johnson, L. A.; Kochhar, A. K.; Kouvelis, P.; McLeavey, D. W.; Melnyk, S. A.; Monden, Y.; Montgomery, D. C.; Nahmias, S.; Narasimhan, S.; Ohno, T.; Orlicky, J.; Peterson, R.; Pinedo, M.; Silver, E. A.; Sipper, D.; Spearman, M. L.; Vollman, T. E.; Whybark, C. e Wight, O.

Todos esses autores atendem aos dois critérios dos elegíveis, em particular todos possuem pelo menos um livro conhecido, a saber: Bayley e Bedworth (1987), Baker (1974), Buffa e Miller (1979), Buffa e Sarin (1987), Burbidge (1975; 1978; 1996), Elmaghraby (1977), Goldratt e Cox (1984), Hax e Candea (1984), Hopp e Spearman (2000), Johnson e Montgomery (1974), Kochhar (1974), Kouvelis e Yu (1997), Melnyk et al. (1985), Monden (1983, 1986), Nahmias (2001), Narasimhan et al. (1985), Ohno (1988), Orlicky (1975), Pinedo (2001; 2006), Pinedo e Xiuli (1999), Silver e Peterson (1985), Sipper e Bulfin (1997), Vollmann et al. (1997) e Wight (1967, 1981).

Passo 6: Determinação da técnica para realizar a votação com a amostra de pesquisadores selecionados e elaboração do questionário.

Como entrevistas, técnicas de observação, análise de gravações e etc. são técnicas inviáveis para esta pesquisa, uma vez que o custo e o tempo necessário para realizá-las são muito altos, é necessário decidir-se entre questionário postal e questionário por e-mail.

Optou-se pelo questionário por e-mail, principalmente em decorrência do baixo custo, alta velocidade de envio do questionário e da maior praticidade para ser respondido o que poderia contribuir para diminuir o número de não-respostas.

O questionário foi elaborado de forma a identificar as seguintes questões:

a) os top-autores em PCP: o entrevistado deveria destacar os dez nomes mais importantes por meio da escolha entre os nomes apresentados ou adicionar novos nomes à lista. $\mathrm{O}$ método de apresentar uma lista preliminar pode ter causado um viés por direcionar as respostas, mas foi a melhor maneira encontrada para aumentar o nível de respostas. Além disso, tentou-se minimizar o direcionamento das respostas, deixando-se espaços para que o entrevistado pudesse inserir novos candidatos à lista;

b) verificar qual o enfoque de pesquisa do entrevistado. Isto pôde ser medido por meio de uma questão contendo alternativas para que o entrevistado assinalasse os enfoques com que possui maior familiaridade, dentre as quatro categorias seguintes: aspectos gerenciais de PCP (conceitos, procedimentos e práticas gerenciais); aplicações de pesquisa operacional no PCP; aplicações de inteligência artificial no PCP; e aplicações de sistemas de informação/tecnologia de informação no PCP;

c) verificar qual a origem da formação acadêmica do entrevistado. Colocou-se uma questão para que ele respondesse em que região tinha obtido seu último título acadêmico dentre estas três: EUA; Europa; e resto do mundo;

d) tempo de atuação em PCP do entrevistado: menor ou igual a 5 anos; entre 5 e 15 anos; entre 15 e 25 anos; e mais de 25 anos; e

e) influência e impacto dos autores citados pelo entrevistado no PCP: A influência pode ser direta sobre toda a área de PCP (I1); pode ser sobre algum problema específico do PCP (I2) ou pode ser uma influência indireta sobre a área de PCP (I3). Já o impacto pode ser na teoria e pesquisa em PCP (IA); no ensino de PCP (IB); ou na indústria e prática referente ao PCP (IC).

Passo 7: Envio do questionário por e-mail a todos os pesquisadores da amostra selecionada e recebimento e armazenamento das respostas.

Dos 205 e-mails enviados, 43 não chegaram ao seu destino (retornaram). Dos 162 pesquisadores da amostra que receberam o e-mail (amostra corrigida), após três replicações, 70 retornaram com respostas o questionário. Esta taxa de retorno $(70 / 162=43,21 \%)$ pode ser considerada excelente, uma vez que não havia nenhum estímulo extra, por exemplo, sorteio de um prêmio, para os pesquisadores que respondessem ao questionário.

$\mathrm{O}$ passo 8 da pesquisa, que corresponde à apresentação e análise estatística dos resultados, é mostrada nas seções 5 e 6 .

\section{Resultados}

Finalizada a pesquisa com a amostra, foi utilizado o estimador de Horwitz-Thompson para identificação dos principais autores do PCP na visão da população de pesquisadores. 
Neste artigo, o estimador de Horwitz-Thompson (COCHRAN, 1977) é um estimador não viciado para a proporção de votos para o autor $j\left(\tau_{j}\right)$ e pode ser escrito da seguinte forma:

$$
\hat{\tau}_{\pi j}=\sum_{i=1}^{v} \frac{y_{i j}}{\pi_{i}}, j=1, \ldots, J
$$

sendo $v$ o número de unidades distintas na amostra; $y_{i j}=1$ se a unidade $i$ da amostra votou no autor $j ; y_{i j}=0$ caso contrário para $i=1, \ldots, v$ e $j=1, \ldots, J$; e $\pi_{i}$ a probabilidade de que a unidade $i$ seja incluída na amostra, para $i=1, \ldots, \mathrm{v}$.

Sendo $p_{i}$ a probabilidade de que a unidade $i$ seja selecionada, então a probabilidade que a unidade $i$ não seja incluída na amostra de tamanho $n$, quando a amostra é com reposição, é $\left(1-p_{i}\right)^{n}$. Portanto, a probabilidade $\pi_{i}$ de que a unidade $i$ seja incluída na amostra é $1-\left(1-p_{i}\right)^{n}$, $i=1, \ldots, n$.

Deste modo, o estimador de Horvitz-Thompson pode ser escrito da seguinte forma:

$$
\hat{\tau}_{\pi j}=\sum_{i=1}^{v} \frac{y_{i j}}{1-\left(1-p_{i}\right)^{n}}, j=1, \ldots, J
$$

Em que $v$ (número de unidades distintas da amostra corrigida $)=162$ pesquisadores; $n$ (tamanho da amostra corrigida $)=162$ pesquisadores; e $p_{i}$ pode ser definido como o peso do pesquisador $i\left(w_{i}=1,2,3\right.$ ou 4$)$ dividido pela soma do peso de todos os pesquisadores (TIS - Total do Intervalo de Seleção), ou seja, $p_{i}=w_{i} /$ TIS.

Como a pesquisa com a amostra foi baseada apenas nas pessoas cujos e-mails funcionavam, foi necessário determinar o TIS para aqueles da população cujos e-mails funcionavam. Devido à escassez de tempo para testar todos os e-mails da população, decidiu-se realizar uma estimativa do número das pessoas da população cujos e-mails funcionavam (chamada aqui de População Corrigida (PC)) e isso foi feito da seguinte maneira: para cada grupo (grupo 1: pesquisadores com 1 artigo;...; grupo 9: pesquisadores com 9 artigos) foi calculada a porcentagem de e-mails confiáveis na amostra corrigida. Esta porcentagem foi aplicada nos grupos da população para se obter a população corrigida (PC) e os respectivos intervalos de seleção.

Os resultados encontrados foram: $N c\left(\mathrm{n}^{\circ}\right.$ de pessoas da PC) $=335$ pesquisadores; TIS $c=367 ; N=592$ pesquisadores (população); $n=162$ pesquisadores (amostra corrigida); e $\mathrm{v}=162$ pesquisadores distintos.

Portanto, o estimador de Horwitz-Thompson fica:

$$
\hat{\tau}_{\pi j}=\sum_{i=1}^{162} \frac{y_{i j}}{1-\left(1-\frac{w_{i}}{367}\right)^{162}}, j=1, \ldots, J
$$

Na Tabela 5, são mostrados os 10 principais autores do PCP, estimados para a PC, e suas principais obras. A Tabela 6 mostra quais são os principais tipos de impacto e influência que eles tiveram na área de PCP.

Tabela 6. Influência (I1, I2 e I3) e impacto (IA, IB e IC) dos principais autores do PCP.

\begin{tabular}{llcccccccc}
\hline $\mathbf{N}^{\mathbf{0}}$ & \multicolumn{1}{c}{ Autor } & \multicolumn{3}{c}{ Influência } & & \multicolumn{3}{c}{ Impacto } \\
\cline { 3 - 4 } \cline { 8 - 9 } & & $\mathbf{I 1}^{\mathbf{a}}$ & $\mathbf{I 2}^{\mathbf{b}}$ & $\mathbf{I 3}^{\mathbf{c}}$ & & $\mathbf{I A}^{\mathbf{d}}$ & $\mathbf{I B}^{\mathbf{e}}$ & $\mathbf{I C}^{\mathbf{f}}$ \\
\hline 1 & Orlicky, J. & 4 & 7 & 1 & & 3 & 1 & 8 \\
2 & Goldratt, E. & 6 & 4 & 1 & & 0 & 0 & 11 \\
3 & Elmaghraby, S. E. & 7 & 0 & 0 & & 3 & 2 & 0 \\
4 & Baker, K. & 4 & 8 & 1 & & 11 & 1 & 0 \\
5 & Pinedo, M. & 4 & 5 & 2 & & 8 & 0 & 2 \\
6 & Silver, E. A. & 7 & 5 & 1 & & 8 & 4 & 1 \\
7 & Buffa, E. S. & 6 & 1 & 1 & & 4 & 2 \\
8 & Ohno, T. & 3 & 5 & 0 & & 0 & 6 \\
9 & Montgomery,D. C. & 2 & 1 & 1 & & 4 & 0 & 0 \\
10 & Burbidge, J. L. & 1 & 3 & 0 & & 1 & 1 & 1 \\
& & Total & 53 & 50 & 13 & & 51 & 37 & 39 \\
\hline
\end{tabular}

ainfluência direta em toda a área de PCP; binfluência direta em algum problema específico da área de PCP; cinfluência indireta sobre a área de PCP; dimpacto em teoria e pesquisa; impacto em ensino; e fimpacto em indústria e prática.

Tabela 5. Os principais autores do PCP, na visão da PC (população corrigida), e suas principais obras.

\begin{tabular}{clcl}
\hline $\mathbf{N}^{\mathbf{0}}$ & \multicolumn{1}{c}{ Autor } & Votos & \multicolumn{1}{c}{ Principais obras } \\
\hline 1 & Orlicky, J. & 92 & Orlicky (1975); Orlicky e Plossl (1994) \\
2 & Goldratt, E. & 83 & $\begin{array}{l}\text { Goldratt e Cox (1984); Goldratt (1986); Goldratt (1994); Goldratt (1997); Goldratt } \\
\text { (1999); Goldratt et al (2000) }\end{array}$ \\
3 & Elmaghraby, S. E. & 79 & Elmaghraby (1977) \\
4 & Baker, K. & 74 & Baker (1974); Baker (1995) \\
5 & Pinedo, M. & 72 & Pinedo e Xiuli (1999); Pinedo (2001, 2006) \\
6 & Silver, E. A. & 71 & Silver e Peterson (1985) \\
7 & Buffa, E. S. & 66 & Buffa e Miller (1979); Buffa e Sarin (1987) \\
8 & Ohno, T. & 65 & Ohno (1988) \\
9 & Montgomery,D. C. & 59 & Johnson e Montgomery (1974) \\
10 & Burbidge, J. L. & 58 & Burbidge (1975, 1978, 1996) \\
\hline
\end{tabular}


Observa-se, na Tabela 6, que os dois autores mais votados na Tabela 5 (Orlicky e Goldratt), junto com Ohno, foram os que tiveram maior impacto na indústria. Os autores que tiveram mais impacto em teoria e pesquisa foram Baker, Pinedo e Silver. Este último, juntamente com Buffa, foi o que teve mais impacto em ensino na área de PCP, com 4 votos cada. Vale ressaltar que os autores Berry, Vollman, e Whybark não estão entre os dez principais autores indicados, mas foram os que tiveram mais votos (5 votos) no quesito impacto em ensino na área de PCP. A maioria dos autores indicados foi apontada por ter influência direta em toda a área de PCP. dentre eles destacaram-se: Elmaghraby, Silver, Goldratt e Buffa. Os autores que mais se destacaram por terem influência direta em algum problema específico da área de PCP foram: Baker, Orlicky, Pinedo, Silver e Ohno (outro autor que obteve destaque neste item foi Spearman com 6 votos).

Portanto, pode-se concluir que as principais contribuições para a área de PCP não foram daqueles que tiveram influência indireta na área, mas sim daqueles que contribuíram de forma direta, seja em toda a área ou em algum problema específico. Além disso, a maioria dos autores mais votados apresentou maior impacto na teoria e pesquisa.

\section{Análise dos resultados e hipóteses}

Apesar de utilizar o estimador de Horvitz-Thompson para a determinação dos principais autores na seção anterior, as análises desta seção foram realizadas considerando-se os votos simples de cada entrevistado, pois a realização de análises por meio do estimador de HorvitzThompson exigiria a adoção de técnicas extremamente complexas, bem como um índice maior de respostas.

O primeiro fator a ser analisado foi a influência da região dos entrevistados no resultado da eleição dos principais autores do PCP.

Ao contrário do que era esperado, não houve "regionalismo" entre os pesquisadores. Na Tabela 7, pode-se observar que os autores americanos foram os mais votados nas três regiões e, portanto, a hipótese 2 (ver seção 2) deve ser rejeitada.
É importante destacar que os pesquisadores europeus apresentaram o maior índice de respostas, com $61,7 \%$ (29 respostas entre os 47 integrantes da $\mathrm{AE}$ ), os americanos tiveram um índice de respostas de 43,6\% (24 respostas entre os 55 integrantes da AE) e os pesquisadores do Resto do Mundo foram os que apresentaram o menor índice de respostas com apenas 28, 3\% (17 respostas entre os 60 integrantes da AE).

Também é importante ressaltar que não houve um consenso sobre os principais autores do PCP entre os entrevistados das três regiões, pelo contrário, houve muita variação da importância dada aos autores indicados. Por exemplo, Pinedo tem uma importância muito grande para os europeus ( $1^{\circ}$ lugar), mas ele é apenas o $12^{\circ}$ na visão dos americanos e o $8^{\circ}$ para os pesquisadores do resto do mundo.

Além disso, na visão dos pesquisadores do Resto do Mundo, houve um grande destaque para a maior valorização do Baker ( $1^{\circ}$ lugar) e a menor importância dada ao Orlicky. Já para os americanos, notou-se uma grande valorização de Berry e de Vollman provavelmente em função do livro Vollman et al. (1997) do qual Berry é coautor. Whybark também é co-autor deste livro e está na sexta posição na visão dos americanos.

Na Tabela 8, verifica-se que a maioria dos pesquisadores da amostra tinha mais familiaridade com os enfoques de pesquisa operacional e aspectos gerenciais de PCP (conceitos, procedimentos e práticas gerenciais).

Observa-se também, na Tabela 9, que alguns autores podem ser considerados universais para a área de PCP,

Tabela 8. Divisão dos pesquisadores da amostra em função da região e do enfoque de pesquisa.

\begin{tabular}{lcccc}
\hline \multirow{2}{*}{ Região } & \multicolumn{4}{c}{ Enfoques de pesquisa } \\
\cline { 2 - 5 } & Aspectos G. ${ }^{\text {a }}$ & PO $^{\mathbf{b}}$ & IA $^{\mathbf{c}}$ & SI/TI $^{\mathbf{d}}$ \\
\hline Europa & 19 & 23 & 6 & 9 \\
Estado Unidos & 19 & 18 & 3 & 6 \\
Resto do Mundo & 9 & 15 & 5 & 3 \\
Total & 47 & 56 & 14 & 18 \\
\hline
\end{tabular}

${ }^{\mathrm{b}}$ Aspectos gerenciais de PCP (conceitos, procedimentos e práticas gerenciais); ' baplicações de pesquisa operacional (PO) em PCP; ${ }^{c} \mathrm{a}-$ plicações de inteligência artificial (IA) em PCP; e daplicações de sistemas de informação (SI)/tecnologia de informação (TI).

Tabela 7. Classificação dos autores em função da região dos votantes.

\begin{tabular}{|c|c|c|c|c|c|c|c|c|c|}
\hline \multirow[t]{2}{*}{$\mathbf{N}^{\mathbf{o}}$} & \multicolumn{3}{|c|}{ Europa $^{a}$} & \multicolumn{3}{|c|}{ Estados Unidos $^{\text {b }}$} & \multicolumn{3}{|c|}{ Resto do mundo $^{c}$} \\
\hline & Autor & Região & Votos & Autor & Região & Votos & Autor & Região & Votos \\
\hline 1 & Pinedo & EUA & 15 & Orlicky & EUA & 15 & Baker & EUA & 10 \\
\hline 2 & Elmaghraby & EUA & 14 & Berry & EUA & 12 & Goldratt & Israel & 10 \\
\hline 3 & Orlicky & EUA & 13 & Goldratt & Israel & 12 & Buffa & EUA & 10 \\
\hline 4 & Silver. & EUA & 11 & Vollman & Suíça & 12 & Spearman & EUA & 9 \\
\hline 5 & Burbidge & Inglaterra & 10 & Silver & EUA & 11 & Elmaghraby & EUA & 9 \\
\hline
\end{tabular}

a29 pesquisadores; ${ }^{\text {b2 }} 4$ pesquisadores; e ${ }^{\text {c }} 17$ pesquisadores. 
uma vez que são importantes em todos os enfoques, como, por exemplo, Orlicky e Goldratt. O primeiro foi o mais votado em três enfoques. Não houve nenhum autor que se destacou em apenas um enfoque, 2 deles (Baker e Elmaghraby) se destacaram em três e o restante (4 autores) foi indicado em 2 enfoques. Portanto, a hipótese 1 (ver seção 2), de que os pesquisadores tendem a escolher autores relacionados ao seu enfoque de pesquisa deve ser aceita como parcialmente verdadeira, pois, embora alguns autores tenham sido indicados apenas por pesquisadores de mesmo enfoque, os mais votados foram os que possuem importância para mais de um enfoque.

É importante destacar que a lista dos principais autores da área (Tabela 5) reflete o desbalanceamento do número de votantes com relação a cada enfoque de pesquisa (Tabela 8). Por exemplo, na Tabela 9 verificase que Vollman aparece entre os cinco principais autores na visão dos pesquisadores dos enfoques aspectos gerenciais e aplicações de SI em PCP, entretanto, ele não está na lista dos 10 maiores autores da área (Vollman ocupa a $11^{\mathrm{a}}$ posição). Por outro lado, Montgomery (apesar de há muitos anos estar realizando pesquisas em métodos estatísticos) ficou em $11^{\circ}$ lugar na lista dos pesquisadores de PO e de IA, $20^{\circ}$ lugar na lista dos votantes de aspectos gerenciais e em $18^{\mathrm{a}}$ na lista dos pesquisadores em SI, contudo ele está em $9^{\circ}$ lugar na lista dos principais autores da área. Deste modo, a predominância de pesquisadores da área de PO foi responsável pela inclusão do Montgomery na lista da Tabela 5, no lugar do Vollman.

Do mesmo modo, o fato de Pinedo estar em $5^{\circ}$ lugar na Tabela 5 deve-se à importância que ele tem na área de $\mathrm{PO}$ (está em primeiro lugar), visto que ele não obteve muito destaque nas outras áreas.

As Tabelas 10, 11 e 12, apresentam os autores mais votados em função do enfoque de pesquisa para os europeus, americanos e resto do mundo, respectivamente. Em relação à Europa (Tabela 10), notou-se que o primeiro autor para cada enfoque continua o mesmo, entretanto, Goldratt não obteve o mesmo destaque que na Tabela 9. Por outro lado, houve um maior destaque para Buffa e, principalmente, Elmaghraby, já que ele se mostrou importante em todos os enfoques na visão dos europeus.

Tabela 9. Autores mais votados em função do enfoque de pesquisa dos entrevistados.

\begin{tabular}{|c|c|c|c|c|c|c|c|c|}
\hline \multirow[t]{2}{*}{$\mathbf{N}^{\mathbf{o}}$} & \multicolumn{2}{|c|}{ Aspectos G. ${ }^{a}$} & \multicolumn{2}{|c|}{$\mathbf{P O}^{\mathrm{b}}$} & \multicolumn{2}{|l|}{$\mathbf{I A}^{\mathbf{c}}$} & \multicolumn{2}{|c|}{$\mathrm{SI} / \mathbf{T I}^{\mathrm{d}}$} \\
\hline & Autor & Votos & Autor & Votos & Autor & Votos & Autor & Votos \\
\hline 1 & Orlicky & 28 & Pinedo & 29 & Orlicky & 9 & Orlicky & 11 \\
\hline 2 & Goldratt & 23 & Orlicky & 28 & Goldratt & 8 & Goldratt & 10 \\
\hline 3 & Baker & 19 & Elmaghraby & 26 & Burbidge & 7 & Elmaghraby & 9 \\
\hline 4 & Burbidge & 19 & Baker & 25 & Elmaghraby & 7 & Buffa & 8 \\
\hline 5 & Vollman & 19 & Goldratt & 25 & (Baker; Buffa; Pinedo) & 6 & Vollman & 7 \\
\hline
\end{tabular}

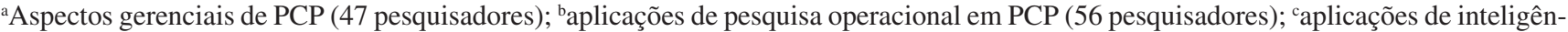
cia artificial em PCP (14 pesquisadores); e daplicações de SI / TI em PCP (18 pesquisadores).

Tabela 10. Autores mais votados em função do enfoque de pesquisa dos europeus entrevistados.

\begin{tabular}{|c|c|c|c|c|c|c|c|c|}
\hline \multirow[t]{2}{*}{$\mathbf{N}^{\mathbf{o}}$} & \multicolumn{2}{|c|}{ Aspectos G. ${ }^{a}$} & \multicolumn{2}{|c|}{$\mathbf{P O}^{\mathbf{b}}$} & \multicolumn{2}{|l|}{$\mathbf{I A}^{\mathbf{c}}$} & \multicolumn{2}{|c|}{$\mathrm{SI} / \mathbf{T I}^{\mathrm{d}}$} \\
\hline & Autor & Votos & Autor & Votos & Autor & Votos & Autor & Votos \\
\hline 1 & Orlicky & 10 & Pinedo & 14 & Orlicky & 5 & Orlicky & 6 \\
\hline 2 & Elmaghraby & 9 & Elmaghraby & 12 & Elmaghraby & 4 & Buffa & 5 \\
\hline 3 & Burbidge & 8 & Orlicky & 10 & Buffa, Burbidge e Pinedo & 3 & Elmaghraby & 5 \\
\hline
\end{tabular}

${ }^{a}$ Aspectos gerenciais de PCP (19 pesquisadores); ${ }^{b}$ aplicações de pesquisa operacional (PO) em PCP (23 pesquisadores); ${ }^{\text {baplicações de }}$ inteligência artificial (IA) em PCP (06 pesquisadores); e caplicações de sistemas de informação (SI)/tecnologia de informação (TI) em PCP (09 pesquisadores).

Tabela 11. Autores mais votados em função do enfoque de pesquisa dos americanos entrevistados.

\begin{tabular}{|c|c|c|c|c|c|c|c|}
\hline \multicolumn{2}{|c|}{ Aspectos G. ${ }^{a}$} & \multicolumn{2}{|c|}{$\mathbf{P O}^{\mathrm{b}}$} & \multicolumn{2}{|l|}{$\mathbf{I A}^{\mathrm{c}}$} & \multicolumn{2}{|c|}{$\mathrm{SI} / \mathbf{T I}^{\mathrm{d}}$} \\
\hline Autor & Votos & Autor & Votos & Autor & Votos & Autor & Votos \\
\hline Orlicky & 13 & Orlicky & 10 & Baker & 3 & Berry & 4 \\
\hline Berry & 12 & Silver & 10 & Berry, Buffa, Burbidge e Goldratt & 2 & Goldratt & 4 \\
\hline Goldratt e Vollman & 10 & Vollman & 9 & & & Orlicky & 4 \\
\hline
\end{tabular}

${ }^{a}$ Aspectos gerenciais de PCP (19 pesquisadores); baplicações de pesquisa operacional (PO) em PCP (18 pesquisadores); ${ }^{c}$ aplicações de inteligência artificial (IA) em PCP (03 pesquisadores); e daplicações de sistemas de informação (SI)/tecnologia de informação (TI) em PCP (06 pesquisadores). 
Conforme ressaltado anteriormente, os europeus foram os responsáveis pelo fato do Pinedo estar em $1^{\circ}$ lugar também na área de PO.

Em relação aos americanos (Tabela 11), é importante realçar a maior importância dada a Berry que se destacou em 3 enfoques e não foi indicado pelos europeus e pelo resto do mundo nas Tabelas 10 e 11 .

A lista dos autores mais votados pelos pesquisadores do Resto do Mundo (Tabela 12) foi a que apresentou maior quantidade de pessoas cujos nomes não estavam entre a lista dos 10 maiores autores da área. Os autores que mais se destacaram foram Goldratt e Spearman que foram indicados para os 4 enfoques, além de Baker e Orlicky que foram indicados para 3 enfoques.

Em relação ao tempo de experiência dos entrevistados, observou-se que a maioria deles tinha entre 5 e 15 anos de experiência na área. Dos 70 entrevistados, apenas 37 responderam a essa questão, conforme explicitado na legenda da Tabela 13.

Por meio da tabela 13, percebe-se que algumas categorias, como por exemplo, a daqueles que possuem menos de 5 anos de experiência na área, possuem poucos representantes ( 2 pessoas) e, portanto, a análise desta variável fica prejudicada. Entretanto, percebe-se que o grupo de pesquisadores mais jovens não deixou de citar os autores de livros clássicos, como Baker, Elmaghraby e Orlicky. Por outro lado, pesquisadores mais experientes (até 25 anos de experiência) não deixaram de citar os autores mais modernos, como por exemplo, Pinedo. Contudo, aqueles que possuem mais de 25 anos de experiência na área deram ênfase aos autores mais antigos, que apesar de terem edições publicadas recentemente, já eram famosos há alguns anos atrás.

Por meio da Tabela 14, verifica-se que a hipótese 3 (ver seção 2) deve ser aceita visto que em alguns casos

Tabela 12. Autores mais votados em função do enfoque de pesquisa dos entrevistados do resto do mundo.

\begin{tabular}{|c|c|c|c|c|c|c|c|}
\hline \multicolumn{2}{|l|}{ Aspectos G. ${ }^{a}$} & \multicolumn{2}{|l|}{$\mathbf{P O}^{\mathrm{b}}$} & \multicolumn{2}{|l|}{$\mathbf{I A}^{\mathrm{c}}$} & \multicolumn{2}{|l|}{$\mathrm{SI} / \mathrm{TI}^{\mathrm{d}}$} \\
\hline Autor & Votos & Autor & Votos & Autor & Votos & Autor & Votos \\
\hline Goldratt, E. & 7 & Goldratt, E. & 9 & Goldratt, E. & 4 & Spearman, M. L. & 3 \\
\hline $\begin{array}{l}\text { Baker, K., Orlicky, J., } \\
\text { e Spearman, M. L }\end{array}$ & 5 & $\begin{array}{l}\text { Spearman, M. L., Baker, K., } \\
\text { Buffa, E. S., Elmaghraby, } \\
\text { S. E. e Orlicky, J. }\end{array}$ & 9 & $\begin{array}{l}\text { Montgomery, D. C., } \\
\text { Orlicky, J. e } \\
\text { Spearman, M. L. }\end{array}$ & 3 & $\begin{array}{l}\text { Baker, K. } \\
\text { Elmaghraby, S. E. } \\
\text { Goldratt, E. } \\
\text { Hopp, W. J. } \\
\text { Ohno, T. }\end{array}$ & 2 \\
\hline
\end{tabular}

${ }^{\mathrm{a}}$ Aspectos gerenciais de PCP (1 pesquisadores); baplicações de pesquisa operacional (PO) em PCP (3 pesquisadores); ${ }^{\mathrm{c}}$ aplicações de inteligência artificial (IA) em PCP (3 pesquisadores); e daplicações de sistemas de informação (SI)/tecnologia de informação (TI) em PCP (2 pesquisadores).

Tabela 13. Classificação dos principais livros dos autores mais votados em função do tempo de pesquisa.

\begin{tabular}{|c|c|c|c|c|c|c|c|c|}
\hline \multirow[t]{2}{*}{$\mathbf{N}^{\mathbf{o}}$} & \multicolumn{2}{|c|}{$\begin{array}{c}\leq 5 \text { anos } \\
(2 \text { entrevistados })\end{array}$} & \multicolumn{2}{|c|}{$\begin{array}{c}\geq 5 \text { e } \leq 15 \text { anos } \\
(17 \text { entrevistados })\end{array}$} & \multicolumn{2}{|c|}{$\begin{array}{c}\geq 15 \text { e } \leq 25 \text { anos } \\
(12 \text { entrevistados })\end{array}$} & \multicolumn{2}{|c|}{$\geq 25$ anos ( 6 entrevistados) } \\
\hline & Autor & Votos & Autor & Votos & Autor & Votos & Autor & Votos \\
\hline 1 & Elmaghraby & 2 & Whybark & 11 & $\begin{array}{l}\text { Elmaghraby, } \\
\text { Orlicky, Pinedo, } \\
\text { Silver e Spearman }\end{array}$ & 6 & Orlicky & 5 \\
\hline 2 & $\begin{array}{l}\text { Bayley, Baker, } \\
\text { Buffa, Bulfin, Ohno, } \\
\text { Orlicky, Pinedo, } \\
\text { Silver e Spearman }\end{array}$ & 1 & Orlicky e Silver & 8 & & & $\begin{array}{l}\text { Buffa, Ohno, } \\
\text { Spearman }\end{array}$ & 4 \\
\hline 3 & & & $\begin{array}{l}\text { Goldratt, Pinedo e } \\
\text { Vollmann }\end{array}$ & 7 & & & $\begin{array}{l}\text { Berry, Goldratt, Hopp } \\
\text { Vollmann, Wight e } \\
\text { Elmaghraby }\end{array}$ & 3 \\
\hline
\end{tabular}

Tabela 14. Algumas comparações na classificação dos autores de um mesmo livro.

\begin{tabular}{|c|c|c|c|c|c|c|}
\hline Livro & $\mathbf{1}^{\circ}$ Autor & Posição & $2^{\circ}$ Autor & Posição & $3^{\circ}$ Autor & Posição \\
\hline Silver e Peterson (1985) & Silver & $6^{\circ}$ & Peterson & $20^{\circ}$ & - & - \\
\hline Johnson e Montgomery (1974) & Johnson & $14^{\circ}$ & Montgomery & $9^{\circ}$ & - & - \\
\hline Vollmann et al. (1997) & Vollman & $11^{\circ}$ & Berry & $16^{\circ}$ & Whybark & $17^{\circ}$ \\
\hline Hax e Candea (1984) & $\operatorname{Hax}$ & $12^{\circ}$ & Candea & $*$ & - & - \\
\hline Morton e Pentico (1993) & Morton & $25^{\circ}$ & Pentico & $*$ & - & - \\
\hline
\end{tabular}

*Autor não recebeu nenhum voto. 
apenas um dos autores foi indicado, como por exemplo, nos livros Hax e Candea (1984) e Morton e Pentico (1993) e, em outros, havia grande diferença nas posições dos autores na lista, como, por exemplo, Silver e Peterson (1985).

\section{Considerações finais}

Apesar da área de PCP ser antiga e possuir uma enorme quantidade de pesquisas já desenvolvidas, ainda não existe um consenso entre os pesquisadores da área sobre quem são os principais autores do PCP.

Dentro deste contexto este trabalho dá um primeiro e importante passo, uma vez que, por meio de uma pesquisa em uma amostra mundial de pesquisadores em PCP, identifica os principais autores (bem como suas obras) da área de PCP. Os 10 principais autores identificados foram: Orlick; Goldratt; Elmaghraby; Baker; Pinedo; Silver; Buffa; Ohno; Montgomery; e Burbidge.

Com relação à influência dos principais autores sobre o PCP, tem-se que a grande maioria dos autores citados foi apontada por ter influência direta na área de PCP, dentre eles destacam-se Elmaghraby; Silver; Goldratt; e Buffa. Os autores que mais se destacaram por terem influência sobre um problema específico do PCP foram Baker; Orlick; Spearman; Pinedo; Silver; e Ohno.

Com relação ao impacto dos principais autores sobre o PCP tem-se que Orlicky, Goldratt e Ohno foram os autores que tiveram maior impacto na indústria. Já na teoria e pesquisa os autores que tiveram mais impacto foram Baker, Pinedo e Silver. Este último, com Buffa, Berry, Vollman e Whybark, foi o autor que mais impactou no ensino na área de PCP.
Esta pesquisa também testa três hipóteses. Com relação à primeira hipótese, ou seja, que os pesquisadores de forma geral tendem a escolher autores relacionados ao seu enfoque de pesquisa, esta deve ser aceita como parcialmente verdadeira, uma vez que embora alguns autores tenham sido indicados apenas por pesquisadores do mesmo enfoque, os mais votados foram os que possuem uma importância para mais de um enfoque. Com relação à segunda hipótese, ou seja, de que existe certo "regionalismo" entre os pesquisadores internacionais, concluiu-se que esta hipótese deve ser rejeitada, uma vez que autores americanos foram os mais votados nas três regiões pesquisadas (Europa, EUA e resto do mundo). Com relação à terceira hipótese, ou seja, de que o fato de um autor participar da elaboração de um livro clássico e bem conceituado não garante que o autor possa ser classificado como um dos mais importantes autores de PCP, concluiu-se que esta hipótese deve ser aceita, uma vez que, em vários casos, livros consagrados como Hax e Candea (1984), Morton e Pentico (1993) tem seus autores em posições bem diferentes com relação à importância para o PCP.

Os principais méritos deste artigo são:

a) detectar, dentre uma infinidade de obras relacionadas ao PCP, quais são as de maior impacto para a área;

b) direcionar os estudos daqueles que têm interesse em aprofundar seus conhecimentos na área de PCP por meio da apresentação dos principais livros da área;

c) valorizar os principais autores da área e dar o primeiro passo na determinação dos grandes mentores do PCP; e

d) demonstrar que a Internet é uma ferramenta muito importante para trabalhos com objetivos similares a este.

\title{
Identification of production planning and control (PPC) top authors by means of a world survey with PPC researchers
}

\begin{abstract}
Although the Production Planning and Control (PPC) area is very old and has a large number of research papers written, it has no agreement regarding who are the PPC top authors. The main aim of this paper is to identify the PPC top authors by means of a survey with PPC researchers. Ten PPC top authors were identified, as well as their influence (direct or indirect or about some specific PPC problem) and impact (in industry or in theory and in research or in PPC teaching) in the PPC area. The main contributions of this paper are: i) identify, among a great number of books regarding PPC, which books have more influence on the PPC area; ii) guide the study of the PPC by means of presenting the main books and authors of PPC; iii) value the PPC top authors, serving as a first step in determining the PPC gurus.
\end{abstract}

Keywords: Production planning and control. Top authors. Global survey. 
BAILEY, J. E.; BEDWORTH, D. D. Integrated production control systems: management, analysis, design. 2. ed. New York, USA: John Wiley \& Sons, 1987.

BAKER, K. R. Introduction to sequencing and scheduling. New York, USA: John Wiley \& Sons Inc., 1974.

Elements of sequencing and scheduling. revised ed. New York, USA: Kenneth R. Baker, 1995.

BONNEY, M. Reflections on production planning and control (PPC). Gestão \& Produção. São Carlos: DEP-UFSCar, v. 7, n. 3, p. 181-207, dezembro de 2000.

BRYMAN, A.; Research methods and organization studies. London: Routledge, 1995.

BUFFA, E. S.; MILLER, J. G. Production - inventory systems. planning and control. 3. ed. Illinois: Richard D. Irwin Inc, 1979.

BUFFA, E. S.; SARIN, R. K. Modern production / operations management. 8. ed. Los Angeles: John Wiley \& Sons, 1987.

BURBIDGE, J. L.; Period batch control. Oxford: Clarendon Press, 1996

The introduction of group technology. London: Heinemann, 1975.

The Principles of Production Control. Estover, Plymouth: MacDonald and Evans Limited, 1978.

BURNS, A. C.; BUSH, R. F. Marketing research. 3. ed. New Jersey: Prentice Hall, 2000.

COCHRAN, W. G. Sampling techniques. 3. ed. New York, USA: John Wiley \& Sons, 1977.

CORREA, H. L.; GIANESE, I. G. N.; CAON, M. Planejamento, programação e controle da produção. 4. ed. São Paulo, SP, Brasil: Editora Atlas, 2001.

CRESWELL, J. W.; Research design - qualitative \& quantitative approaches, London: Sage, 1994.

ELMAGHRABY, S. E. Activity networks: project planning and control by network models. John Wiley \& Sons, june, 1977.

GOLDRATT, E. M.; COX, J. The goal. New York, USA: Creative Output BV, 1984.

GOLDRATT, E. M. The race. New York, USA: North River Press Publishing Corporation, 1986.

It's not luck. New York, USA: North River Press Publishing Corporation, 1994.

Critical chain. New York, USA: North River Press Publishing Corporation, 1997.

Theory of constraints. New York, USA: North River Press Publishing Corporation, 1999.

GOLDRATT, E. M.; SCHRAGENHEIM, E.; PTAK, C. A. Necessary but not sufficient. New York, USA: North River Press Publishing Corporation, 2000.

HANSEN, M. H.; HURWITZ, W. N. On theory of sampling from finite population. New York, USA: Ann. Amer. Stat. Assoc., 1943.

HAX, A. C.; CANDEA, D. Production and inventory management. Englewood Cliffs, N. J.: Prentice Hall, 1984.

HOPP, W. J.; SPEARMAN, M. L. Factory physics: foundations of manufacturing management. 2. ed. Boston: Irwin/McGraw-Hill, 2000.

HUCZYNSKI, A. A. Management gurus: what makes them and how to become one. London: Routledge, 1993.
JOHNSON, L. A.; MONTGOMERY, D. C. Operations research in production planning, scheduling, and inventory control. New York: John Wiley \& Sons, 1974.

KOCHHAR, A. K. Development of Computer-Based Production Systems. London: Edward Arnold, 1974.

KOUVELIS, P.; YU, G. Robust discrete optimization and its applications (nonconvex optimization and its applications, V. 14). 1. ed. Kluwer Academic Publishers, 1997.

MELNYK, S. A. et al. Shop floor control - american production and inventory control society. Illinois: Dow Jones-Irwin, 1985.

MONDEN, Y. Toyota production system. Norcross, GA: Industrial Engineering and Management Press (Institute of Industrial Engineers), 1983.

MONDEN, Y. (ed.) Applying JIT. Norcross, GA: Industrial Engineering and Management Press (Institute of Industrial Engineers), 1986.

MORTON, T. E.; PENTICO, D. W. Heuristic scheduling systems: with applications to production systems and project management. New York, USA: John Wiley \& Sons, Inc. 1993.

NAHMIAS, S.; Production and operations analysis. Boston: McGraw-Hill/Irwin, 2001.

NARASIMHAN, S.; MCLEAVEY, D. W.; BILLINGTON, P. Production planning and inventory control. 2. ed. Englewood Cliffs, New Jersey: Prentice Hall, 1985.

OHNO, T. Toyota production system: beyond large-scale production. New York, USA: Productivity Press, 1988.

ORLICKY, J.; Material requirements planning - the new way of life in production and inventory management. New York: McGraw-Hill, 1975.

ORLICKY, J.; PLOSSL, G. W. Orlicky's material requirements planning. 2. ed. New York, USA: McGraw-Hill Trade, 1994.

PINEDO, M. Scheduling: theory, algorithms, and systems. 2. ed. New York, USA: Prentice Hall, 2001.

Planning and scheduling in manufacturing and services. Springer, 2006.

PINEDO, M.; XIULI, C. Operations scheduling with applications in manufacturing and services. New York, USA: Irwin/ McGraw-Hill. 1999.

REA, L. M.; PARKER, R. A. Designing and conducting survey research - a comprehensive guide. New York, USA: JosseyBass Inc., 1997.

SILVER, E. A.; PETERSON, R. Decision systems for inventory management and production planning. New York, USA: John Wiley \& Sons, 1985.

SIPPER, D.; BULFIN, R. Production: Planning, Control and Integration. New York, USA: McGraw-Hill, 1997.

SLACK, N.; CHAMBERS, S.; JOHNSTON, R. Administração da produção. 2 ed. São Paulo: Editora Atlas S. A., 2002.

VOLLMANN, T. E.; BERRY, W .L.; WHYBARK, C. Manufacturing planning and control systems. 4. ed. New York, USA: Irwin, 1997.

WIGHT, O. Production and inventory control - principles and techniques. Englewood Cliffs, N.J., USA: Prentice-Hall,, 1967. . Manufacturing resource planning: MRP II - unlocking america's productivity potential. New York, USA: Oliver Wight Limited Publications, Inc. 1981. 


\section{Sobre os autores}

\section{Flávio Cesar Faria Fernandes}

Escola de Engenharia de São Carlos, Universidade de São Paulo - USP,

Escola Politécnica, Universidade de São Paulo - USP,

Departamento de Engenharia de Produção, Universidade Federal de São Carlos - UFSCar,

Rod. Washington Luís, Km 235, CEP 13565-905 São Carlos, SP, Brasil,

e-mail: dfcf@ power.ufscar.br

\section{Fábio Azeka}

Engenharia de Produção, Universidade Federal de São Carlos - UFSCar, Rod. Washington Luís, Km 235, CEP 13565-905 São Carlos, SP, Brasil,

e-mail: fabioazeka@uol.com.br

\section{Maria Cecília Mendes Barreto}

Estatística, The University of Notthinghan,

Estatística e Experimentação Agronômica, Escola Superior de Agricultura Luiz de Queiroz - USP,

Estatística, Instituto de Matemática e Estatística da USP,

Departamento de Estatística, Universidade Federal de São Carlos,

Rod. Washington Luís, Km 235, CEP 13565-905, São Carlos, SP, Brasil

e-mail: cbarreto@power.ufscar.br

\section{Moacir Godinho Filho}

Departamento de Engenharia de Produção, Universidade Federal de São Carlos, UFSCar, Rod. Washington Luís, Km 235, CEP 13565-905, São Carlos, SP, Brasil,

e-mail:moacir@dep.ufscar.br 
